\title{
FPGA Based Implementation of Low Complex Adaptive Speckle Suppression Filter for B-mode Medical Ultrasound Images
}

\author{
R. Bharath, Vivek Akkala, P. Rajalakshmi, and Punit Kumar
}

\begin{abstract}
Speckles are considered as noise, which masks the fine information present in B-mode ultrasound images. Speckles appears as small snakes and dense granular like structures which has serious impact on visual perception of an image. Adaptive filter based on local statistics of an image is used to enhance the image by suppressing the noise. Adaptive speckle suppression filter enhance the image by reducing the variance between intrapixel intensities in homogeneous regions and preserving variance across interpixel intensities across the nonhomogeneous regions. In this paper, we implemented low complex adaptive speckle suppression filter on FPGA based kintex7 board. The performance of the filter is evaluated by plotting the pixel variations of original image with filtered image of an ultrasound phantom. The results show that proposed algorithm can be implemented on mobile ultrasound platforms due to $50 \%$ less computations needed per pixel compared to traditional adaptive speckle suppression algorithms, which aids better diagnosis for healthcare.
\end{abstract}

Index Terms-Adaptive filter, FPGA, Image enhancement, Multiplicative noise, Speckle.

\section{INTRODUCTION}

Ultrasound Medical Imaging is the widely used noninvasive imaging technology which uses safe nonradioactive sound waves to visualize the internal body structures like kidney, liver, tumors, heart, etc. Ultrasound devices are doctor friendly due to its unique real time imaging capability compared to Magnetic Resonance Imaging (MRI), Computed Tomography (CT) and Positron Emission Tomography (PET) technologies. Ultrasound offers cheap and fast diagnosis reducing the time to start medication at causality. Medical ultrasound sustains the competition from MRI, CT since they are considered to be golden standard, due to its inexpensive setup and fast diagnosis.

Recent developments in computing platforms like Field Programmable Gate arrays (FPGA), Digital Signal Processors (DSP) has brought down the size of ultrasound machine to portable level which is suitable for point of care applications [1]. Unlikely, ultrasound images suffers from a special type of noise called speckles [2] which also appears in synthetic aperture radar and active radar images [3]. Speckles appears as small granules and worm like structures in the ultrasound image which masks the finer information of tissues, cells etc. Speckle suppression will help the sonographer to diagnose the patients with confidence.

Speckle is an artifact for image enhancement algorithms like edge detection and segmentation tasks, but speckles has

*This work is supported by IIT Hyderabad under 'IU-ATC' project Authors are with Department of Electrical Engineering, Indian Institute of Technology Hyderabad, Hyderabad, India, email: $\{$ ee13p0007, ee12m1041, raji, punit at iith.ac.in\}. to be preserved for organ detection, tissue tracking and computer aided diagnosis. The ultrasound phantom image and corresponding canny edge detection of ultrasound phantom image is shown in Fig. 1. The spurious edges are resulted in contours due to presence of speckle noise in the ultrasound image. Speckles can be suppressed at the RF receiver side by using different compounding techniques [4], [5]. These compounding techniques can be implemented at a cost of hardware complexity, which cannot be offered by portable ultrasound devices. Speckle suppression can also be done by applying image filtering techniques on the ultrasound image [6], [7].

Speckle suppression and information preservation in images is divergent. Speckle suppression comes at a cost of loosing the information in image. The image quality assessment parameters [8] like mean square error (MSE), peak signal to noise ratio (PSNR) and mean structural similarity index (SSIM) will not able to evaluate the performance of the filter as it compares the filtered image with noise affected ultrasound image which is already corrupted with noise. Image quality assessments says to what extent filtered image is deviated from ultrasound image. The performance of the filter is visualized by plotting the pixel variations of original image with filtered image.

The speckle noise give rise to high variations in inter and intrapixels. The solution to speckle noise in this paper is seen as reducing the intrapixel variations in homogeneous regions and preserving the edges simultaneously. In adaptive speckle suppression filters [9]-[12], each pixel is resolved by knowing the local statistics pertaining to that pixel, increasing number of computations per pixel. Low complex adaptive speckle suppression filter reduces the number of computations by dividing the image into small blocks, as-

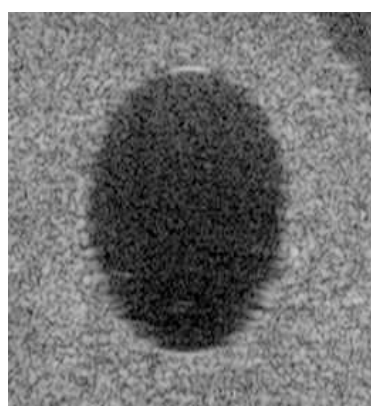

(a)

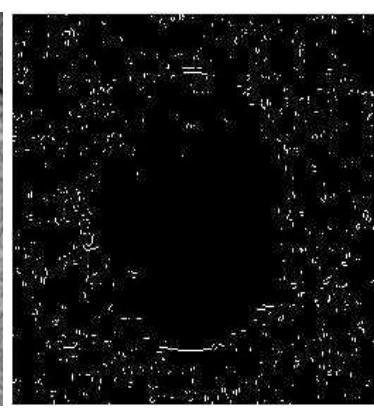

(b)
Fig. 1. (a) Ultrasound phantom image. (b) Canny edge detection. 
suming local statistics of all pixels remains same to that of block it belongs. The pixel is resolved by knowing the local statistics of the block in which it is residing, reducing the number of overall computations required per pixel.

The rest of the paper is organized in the following way, section II gives the statistical behavior and modeling of speckles. Section III gives the description of the low complex adaptive speckle suppression algorithm. Section IV discusses the FPGA implementation of the algorithm. Results of the filter is discussed in section V and section VI concludes the paper.

\section{MATHEMATICAL MODELING OF SPECKLES}

Mathematically speckle noise can be modeled as sum of large number of complex phasors which results in constructive and destructive interference at the receiver side [13]. The constructive interference leads to bright spots and destructive interference leads to dark spots appearing similar to dense salt and pepper like noise [14] in the image.

$$
I(m, n)=\sum_{1}^{p} a_{p}(m, n) e^{j \varphi_{p}(m, n)}
$$

$p$ is a positive integer which is generally considered very large, $a_{p}$ and $\varphi_{p}$ are amplitudes and phases of scattered echoes from tissues. $I(m, n)$ represents intensity of RF signal at $(m, n)$ spatial location of scan plane.

The multiplicative model for envelope detection $J(m, n)$ of RF signal is given by

$$
J(m, n)=(P(m, n) * I(m, n)) N_{\times}(m, n)
$$

where the multiplicative noise $N_{\times}(m, n)$ is sample wise independent of past, future samples and uncorrelated to the image pixel value $I(m, n)$ and $P(m, n)$ is the point spread function (PSF) of the ultrasound imaging system. $(m, n)$ represents the spatial position of pixel in the image. The log transformation, which is used to compress the dynamic range of envelope detected data in ultrasound imaging system will modify the multiplicative model into an additive model.

$$
J(m, n)=(P(m, n) * I(m, n))+N_{+}(m, n)
$$

$N_{+}(m, n)$ is the additive noise term dependent on image pixel value.

Speckle behavior in ultrasound image is better visualized by plotting the histogram of small portion of homogeneous regions. Fig. 2 is an ultrasound phantom image with red and blue boxes indicating the constant reflectivity regions. Fig. 3(a) and Fig. 3(b) represents the histogram of homogeneous regions indicated with blue and red regions of Fig. 2. Homogeneous regions are those which have same intensity distribution. Ideally the histogram of homogeneous region should be of single spike, but the histogram follows some random distribution [15] due to speckle noise.

In [12], it is shown that there is a linear relationship between mean and variance of the speckle patterns in an

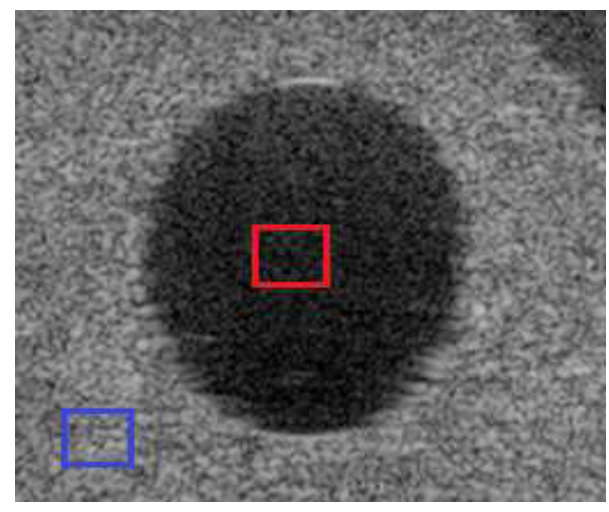

Fig. 2. Ultrasound phantom image.

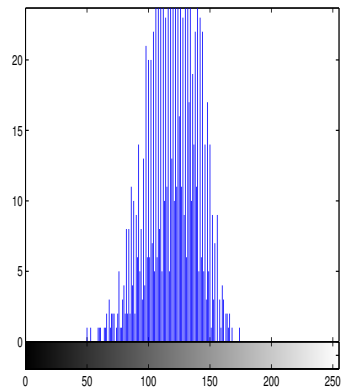

(a)

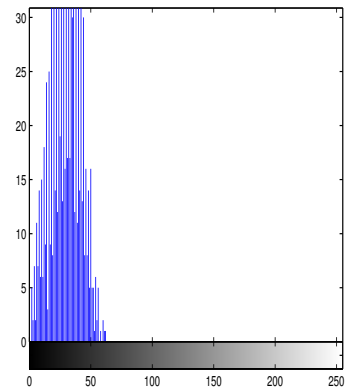

(b)
Fig. 3. Histogram of region indicated in (a) blue color box. (b) red color box.

ultrasound image. The mean and variance of speckle patterns is computed for variable block sizes at different pixel locations of an image.

$$
\sigma_{m, n}^{2}=K \mu_{m, n}
$$

$\sigma^{2}$ and $\mu$ are the variance and mean at $(m, n)$ pixel location, $\mathrm{K}$ is a proportional constant. In [15] it is shown that speckle noise in ultrasound image will follow FisherTippet distribution. Final ultrasound image is modeled to fit it into the equation (4). The signal dependent noise model [12] of an ultrasound image is given by

$$
J=I+\sqrt{I} * N
$$

where $J$ is the observed signal, $I$ is noise free signal and $N$ is noise.

\section{LOW COMPLEX ADAPTIVE SPECKLE SUPPRESSION FILTER DESIGN}

The objective of the low complex adaptive speckle suppression filter is to reduce the number of computations required per pixel for speckle suppression by doing block processing assuming invariant speckle behavior in that particular block. This algorithm first divides the image into fixed size blocks which termed as homogeneous and nonhomogeneous regions based on image statistics of the block. The 
homogeneity of the block is decided by computing variance to mean ratio of pixels present in that block.

$$
\begin{aligned}
\mu & =\left(1 / W^{2}\right) \sum_{m=-W / 2}^{W / 2} \sum_{n=-W / 2}^{W / 2} I(i-m, j-n) \\
\sigma^{2} & =\left(1 / W^{2}\right) \sum_{m=-W / 2}^{W / 2} \sum_{n=-W / 2}^{W / 2}(I(i-m, j-n)-\mu)^{2}
\end{aligned}
$$

Mean $(\mu)$ and variance $\left(\sigma^{2}\right)$ are computed for $\mathrm{WxW}$ block size, consisting of $W^{2}$ pixels, $(i, j)$ represents the spatial location of pixel in the image.

The high variance of block indicates that region is in nonhomogeneous region. High variance will result, if there is a boundary in that block. The low variance indicates there are no boundaries. If variance/mean $\left(\sigma^{2} / \mu\right)$ is greater than threshold then it is said to be in nonhomogeneous region else it is said to be in homogeneous region. The selection of block size is based on statistics of speckle noise. The local statistics of particular block is obtained by computing average and standard deviation of $\sigma^{2} / \mu$ of 10 windows of different sizes each coming from homogeneous region [16].

The average and standard deviation of $\sigma^{2} / \mu$ for different windows of phantom image is shown in Fig. 4. The vertical bar indicates the standard deviation of $\sigma^{2} / \mu$. The low standard deviation $\sigma^{2} / \mu$ of a particular window indicates it can approximate the speckle behavior of a region. $7 \times 7$ and $11 \times 11$ block size has low standard deviation as shown in the Fig. 4. 11x11 is selected as standard grid size as it reduces the number of computations required per pixel further when compared to $7 \times 7$.

The homogeneity of the block is tested with its local statistics, if $\sigma^{2} / \mu$ of the block is greater than or equal to threshold $\left(T_{h}\right)$, which is the sum of average and standard deviation of $\sigma^{2} / \mu$ of $11 \times 11$ block then that block is said to be nonhomogeneous region and need to be resolved

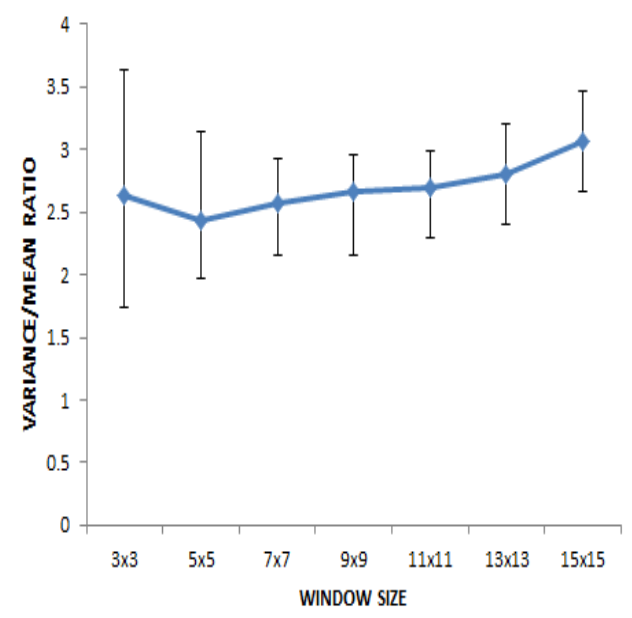

Fig. 4. Local statistics of ultrasound phantom image. else the region is in homogeneous region and mean filter is applied to smooth the region. The computed threshold for ultrasound phantom image for $11 \times 11$ block is 3 . In nonhomogeneous region, there exists two regions and mean filter is not applied which has a property of delineating the edges, so median filter which has the property of preserving the edges is applied.

\section{A. Mean filter}

The mean filter [14] has smoothing effect on the image. It smoothens the high frequency components such as textures and speckles. The $3 \times 3$ convolution template used in homogeneous region to suppress the speckles is shown in Fig. 5. The mean filter will replace original pixel intensity at $I(i, j)$ with average of neighboring $3 \times 3$ pixel intensities.

$$
I(i, j)=(1 / 9) \sum_{m=-1}^{1} \sum_{n=-1}^{1} I(i-m, j-n)
$$

\section{B. Median filter}

Median filter [14] is a nonlinear filter removes the outliers. Median filter will replace the original pixel with median of the $3 \times 3$ surrounding pixels, thus eliminating extremum of the pixels. The extremum pixels are generally contributed by speckles. The median filter has the property of suppressing speckles without delineating the edges.

The low complex adaptive speckle suppression filter decides which filter has to use depending on the local statistics of the image. The flow chart of low complex adaptive speckle suppression filter is shown in Fig. 6.

The adaptive speckle suppression filters based on image local statistics has been discussed in [9]-[12]. For evaluating the performance of our low complex adaptive speckle suppression filter, we choose Lee [9] and Frost [10] filters for comparison.

\section{Lee filter}

Lee filter is based on the local statistics of the pixel. The generalized equation for Lee filter is given by

$$
\hat{I}(m, n)=G(\mu)+K(I(m, n)-\mu)
$$

$G$ is a linear function acting on the local mean and $K$ is the variable gain which depends upon the local statistics of

\begin{tabular}{|c|c|c|}
\hline 1 & 1 & 1 \\
\hline 1 & 1 & 1 \\
\hline 1 & 1 & 1 \\
\hline
\end{tabular}

Fig. 5. Convolution template for mean filter. 
the image. $\hat{I}(m, n)$ is the filtered pixel value of $I(m, n)$. The Lee filter has the property of smoothing the image in homogeneous regions and leaving the pixels unaltered in nonhomogeneous region.

\section{Frost filter}

Frost filter uses exponential kernel which adapts to the local statistics of pixel which is given by

$$
\hat{I}_{f}=\sum_{r \in \eta} h_{r} I_{r}
$$

where

$$
\begin{gathered}
h_{r}=\frac{\exp \left(-K C_{q}^{2} d_{q, r}\right)}{\sum_{r \in \eta} \exp \left(-K C_{q}^{2} d_{q, r}\right)} \\
C_{q}^{2}=\frac{\sigma_{I}^{2}(m, n)}{\bar{I}(m, n)}
\end{gathered}
$$

$d_{q, r}$ is the euclidean distance between the spatial coordinates $q$ and $r$. The value $K$ is chosen adaptively from the local statistics of the image. $\sigma_{I}^{2}(m, n)$ and $\bar{I}(m, n)$ represents local variance and mean of squared image. Frost filter performs similar to mean filter for the pixels at homogeneous region and the pixels across the regions are unaltered. Low complex adaptive filter operates similar to Lee and Frost filter by performing mean filter in homogeneous region and unaltering the pixels at boundaries by operating median filter. The difference between Lee, Frost and proposed adaptive filter is former acts on local statistics of pixels where later acts by block basis.

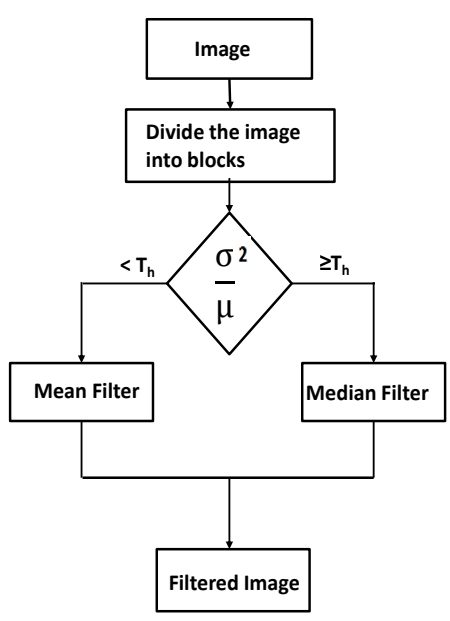

Fig. 6. Flow chart for low complex adaptive speckle suppression filter.

\section{FPGA IMPLEMENTATION OF LOW COMPLEX ADAPTIVE SPECKLE SUPPRESSION FILTER}

Low complex adaptive speckle suppression filter based on local statistics of an image is implemented on FPGA based kintex7 platform [17]. The architecture of proposed low complex adaptive speckle suppression filter is shown in Fig 7.

It mainly consist of mean value generator, variance value generator, comparator, median filter, mean filter, four RAMs, grid address generator and filter address generator. Out of four RAMs, two RAMs are of size $484 \times 484$ while other two RAMs are of size $11 \times 11$. The first $484 \times 484$ RAM is used to store the image. The grid address generator first generates the addresses to store raw image into the first RAM, then it generates address for each $11 \times 11$ sector of image. The data corresponding to the address generated are taken by the mean value generator and variance value generator. Simultaneously, same data is stored in the two 11x11 RAMs. The mean and variance value generator generates the mean and variance value, which is passed to comparator. Comparator compares the $\sigma^{2} / \mu$ to a threshold $\left(T_{h}\right)$ value 3 for $11 \times 11$ block from Fig. 4. If the value is equal or greater than $T_{h}$, then it enables median filter otherwise it enables the mean filter. Once either median or mean filter is enabled, it starts processing each pixel values stored in the first 11x11 RAM using $3 \times 3$ kernel and the corresponding values are stored in second 11x11 RAM. The addresses for processing pixel values stored in the first 11x11 RAM and stores processed pixels in other $11 \times 11$ RAM are generated by filter address generator.

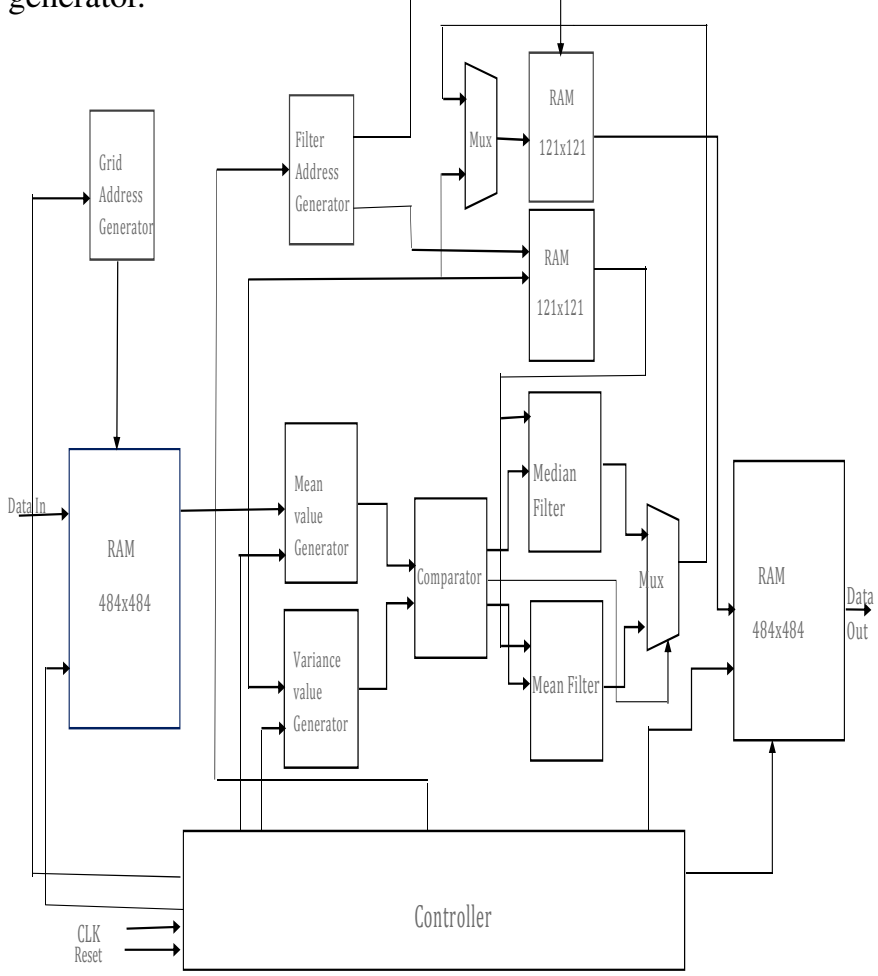

Fig. 7. Hardware architecture of low complex adaptive filter for FPGA kintex7. 
TABLE I

\section{DEVICE UTILIZATION SUMMARY}

\begin{tabular}{|l|l|}
\hline Slice Logic Utilization & \\
\hline Number of Slice Registers & 452 out of $4076000 \%$ \\
Number of Slice LUTs & 841 out of $2038000 \%$ \\
Number used as Logic & 805 out of $2038000 \%$ \\
Number used as Memory & 36 out of $640000 \%$ \\
Number used as SRL & 36 \\
\hline Slice Logic Distribution & \\
\hline $\begin{array}{l}\text { Number of LUT Flip Flop pairs } \\
\text { used }\end{array}$ & 938 \\
Number with an unused Flip Flop & 486 out of $93851 \%$ \\
Number with an unused LUT & 97 out of $93810 \%$ \\
Number of fully used LUT-FF pairs & 355 out of $93837 \%$ \\
Number of unique control sets & 36 \\
\hline IO Utilization & \\
\hline Number of IOs & 19 \\
Number of bonded IOBs & 0 out of $4000 \%$ \\
\hline Specific Feature Utilization & \\
\hline Number of Block RAM/FIFO & 129 out of $44528 \%$ \\
Number using Block RAM only & 129 \\
Number of DSP48E1s & 4 out of $8400 \%$ \\
\hline
\end{tabular}

TABLE II

PERFORMANCE ANALYSIS

\begin{tabular}{|l|l|l|l|}
\hline Filter & MSE & PSNR & SSIM \\
\hline Frost & 24.2302 & 34.32127 & 0.8134 \\
Lee & 53.9054 & 30.8485 & 0.4478 \\
ADF & 4.7309 & 41.4154 & 0.953 \\
\hline
\end{tabular}

Once processing of all pixel values stored in the first $11 \times 11$ RAM is over, the corresponding processed values stored in second $11 \times 11$ RAM is written into second $484 \times 484$ RAM at the same address from where they were taken from the first $484 \times 484$ RAM. The above mentioned process is repeated for the processing of whole image which is stored in the first $484 \times 484$ RAM and the processed image gets stored in the second $484 \times 484$ RAM. The controller is responsible for controlling and synchronizing all the processes. The device utilization summary for FPGA kintex7 board is shown in Table I. The device uses only $0.974 \%$ of slice logic of kintex 7 board which is useful in reducing the area overhead when implemented in Application Specific Integrated Circuit (ASIC).

\section{RESULTS}

The results are interpreted by plotting the pixel variations of single column of low complex adaptive speckle filtered (ADF) image with ultrasound phantom (USP) image. The ADF image is shown in Fig. 8. The pixel intensities of $175^{\text {th }}$ column of Fig. 1(a) with respect to Fig. 8 is shown in Fig 9. From Fig. 9 it is clear that local extremum pixels are smoothed to the local mean. The fall and rise at 50 and 200 pixel indicates edge regions of the image. From the Fig. 9 it is observed that the edges are preserved as red line follows the blue line which

is a result of median filter at that region. The depths of peaks and valleys are significantly reduced which is commonly contributed by speckle noise due to smoothing effect of the mean filter.

Fig. 10 and Fig. 11 represents smoothing of local pixel intensities in Frost and Lee filters respectively. Performance of the low complex speckle suppression adaptive filter is compared with Frost and Lee filter by plotting the pixel variations on the same graph which is shown in Fig. 12 and Fig. 13 respectively. The performance of low complex adaptive speckle suppression filter exactly coincides with Frost filter. The low complex adaptive speckle suppression filter performs better than Lee filter where intra pixel variations are less compared to Lee filter. The image quality assessment metric including MSE, PSNR and SSIM for the filters is shown in Table II. Low complex adaptive filter has low MSE, high PSNR and high SSIM compared to Lee and Frost filters, which is desirable. The error difference between Frost and $\mathrm{ADF}$ is low as pixel variations of both filters coincide as shown in Fig. 12.

Low complex adaptive filter took 30 computations per pixel compared to other filters whose computation per pixel is approximately 60 resulting $50 \%$ computational efficiency. Implementation of Low complex adaptive speckle suppression filter in kintex7 took $2.31 \mathrm{~ms}$ when operated at $100 \mathrm{MHz}$ frequency for an ultrasound image of dimension $484 \mathrm{x} 484$.

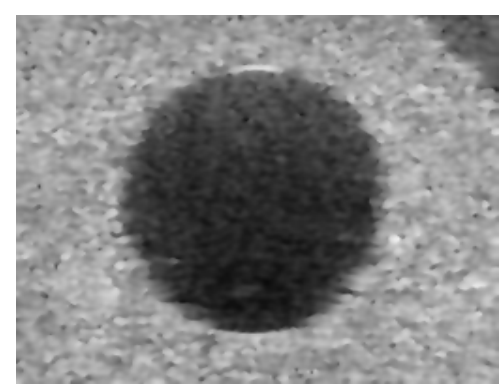

Fig. 8. ADF image.

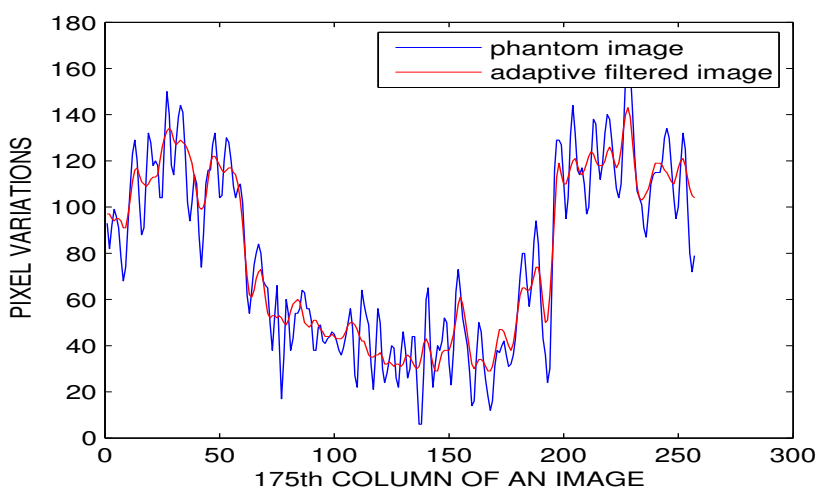

Fig. 9. Pixel variations: USP Vs ADF. 


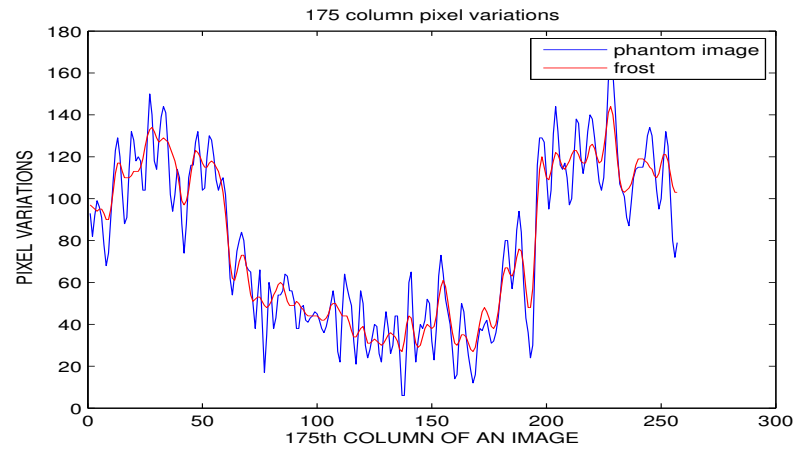

Fig. 10. Pixel variations: USP Vs FROST filtered image.

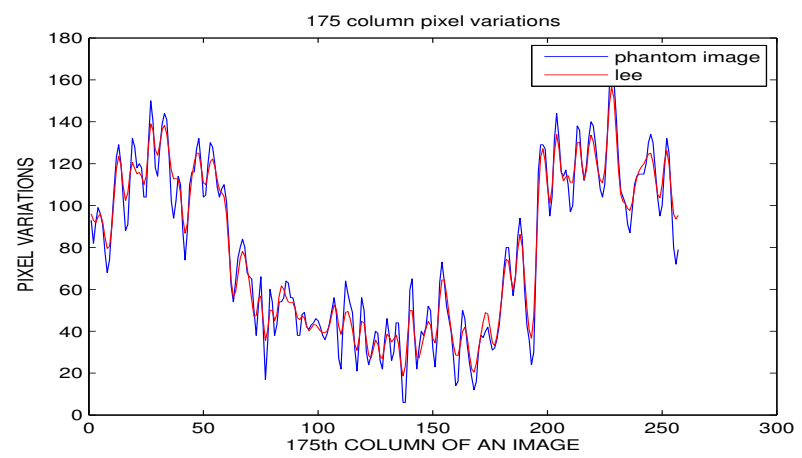

Fig. 11. Pixel variations: USP Vs LEE filtered image.

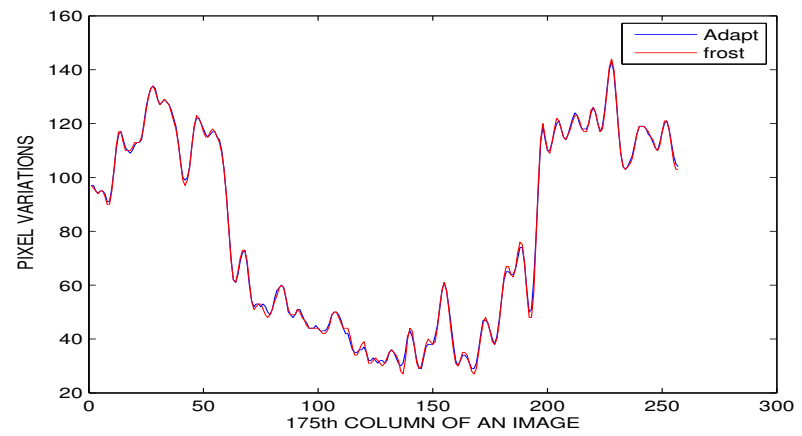

Fig. 12. Pixel variations: ADF Vs FROST filter.

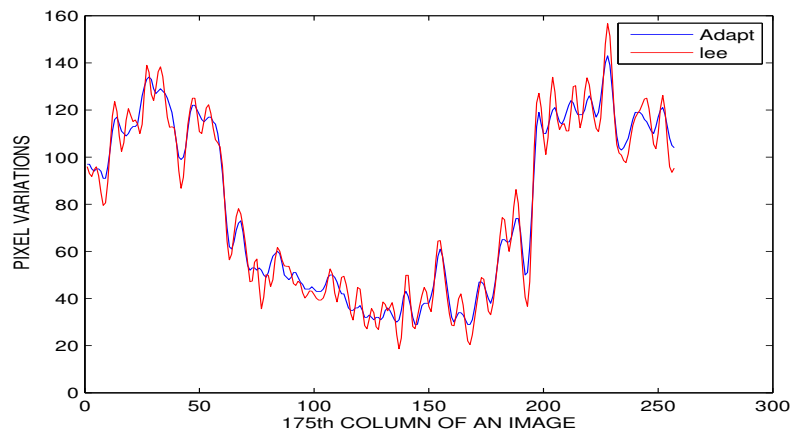

Fig. 13. Pixel variations: ADF Vs LEE filter.

\section{CONCLUSION}

The low complex adaptive speckle suppression algorithm provides despeckling technique to suppress speckle noise with reduced number of computations required per pixel. The number of computations required per pixel is significantly reduced by dividing the image into blocks and processing the pixels based on local statistics of the block where pixel is residing. The low complex adaptive speckle suppression algorithm is implemented on FPGA kintex7 board which can be the computing platform for portable ultrasound system that comes with limiting computational capability. The proposed low complex adaptive speckle suppression algorithm performs similar to Frost and Lee filters with 50\% less in computations producing enhanced images offering better diagnosis for healthcare.

\section{REFERENCES}

[1] Akkala Vivek, P. Rajalakshmi, Punit Kumar, and Uday B. Desai. "FPGA based ultrasound backend system with image enhancement technique." In Biosignals and Biorobotics Conference: Biosignals and Robotics for Better and Safer Living (BRC), 5th ISSNIP-IEEE, pp. 1-5. IEEE, 2014.

[2] J. G. Abbott and F. L. Thurstone, "Acoustic speckle: Theory and experimental analysis", Ultrason. Imaging, vol. 1, pp. 303-324, 1979.

[3] Cheng Hua and Tian Jinwen, "Speckle Reduction of Synthetic Aperture Radar Images Based on Fuzzy Logic", First International Workshop on Education Technology and Computer Science, pp. 933937, 2009

[4] M. Berson, A. Ronan and L. Pourcelat, "Compound scanning with an electrically steered beam", Ultrason. Imag., vol. 3, pp. 303-308, 1981.

[5] P.A. Magnin, O.T. von Ram, and F. L. Thurstone, "Frequency compounding for speckle contrast reduction in phased array images", Ultrason. Imag., vol. 4, pp. 267-281, 1982.

[6] J. I. Koo and S. B. Park, "Speckle reduction with edge preservation in medical ultrasonic images using a homogeneous region growing mean filter", Ultrason. Imag., vol. 13, pp. 211-237, 1991.

[7] G. Castellini, D. Labate, L. Masotti, E. Monnini and S. Rocchi, "An adaptive Kalman filter for speckle reduction in ultrasound images", J. Nucl. Med. Appl. Sci., pp. 208-213, 1988.

[8] Z. Wang, A. C. Bovik, H. R. Sheikh and E. P. Simoncelli, "Image quality assessment: From error visibility to structural similarity", IEEE Trans. Image Process., vol. 13, no. 4, pp. 600612, 2004.

[9] J. S. Lee, "Digital image enhancement and noise filtering by use of local statistics", IEEE Trans. Pattern Anal. Mach. Intell., vol. PAMI-2, no. 2, pp. 165168 , Mar. 1980.

[10] V. S. Frost, J. A. Stiles, K. S. Shanmugan and J. C. Holtzman, ”A model for radar images and its application to adaptive digital filtering of multiplicative noise", IEEE Trans. Pattern Anal. Mach. Intell., vol. PAMI-4, no. 2, pp. 157166, Mar. 1982.

[11] D. T. Kuan, A. A. Sawchuk, T. C. Strand and P. Chavel, "Adaptive noise smoothing filter for images with signal-dependent noise", IEEE Trans. Pattern Anal. Mach. Intell., vol. PAMI-7, no. 2, pp. 165177, Mar. 1985.

[12] T. Loupas, W. N. McDicken and P. L. Allan, "An adaptive weighted median filter for speckle suppression in medical ultrasonic images", IEEE Trans. Circuits Syst., vol. 36, no. 1, pp. 129135, Jan. 1989.

[13] J. W. Goodman, "Statistical properties of laser speckle patterns", in Laser Speckle and Related Phenomena, J. C. Dainty, Ed. Berlin, Germany: Springer-Verlag, pp.975, 1984.

[14] Gonzalez, R.C., Woods, R.E., " Digital Image Processing”, 2nd Ed. Prentice Hall, New Jersey, 2002.

[15] H. H. Arsenault and G. April, "Properties of speckle integrated with a finite aperture and logarithmically transformed", J. Opt. Soc. Amer., vol. 66, no. 11, pp. 11601163, Nov. 1976.

[16] Karaman M., Kutay M., Bozdagi G.,"An adaptive speckle suppression filter for medical ultrasonic imaging", IEEE Trans. Med. Imaging, 14(2):283-292, 1995.

[17] Standard [online]. Available: http://www.xilinx.com productssilicondevices/fpga/kintex-7. 